\title{
SOME INDECOMPOSABLE POLYHEDRA
}

\author{
David Yost
}

\begin{abstract}
We complete the classification, in terms of decomposability, of all combinatorial types of polytopes with 14 or fewer edges. Recall that a polytope $P$ is said to be decomposable if it is equal to a Minkowski sum $Q+R=\{q+r: q \in Q, r \in R\}$ of two polytopes $Q$ and $R$ which are not similar to $P$. Our main contribution here is to consider the 42 types of polyhedra with 8 faces and 8 vertices. It turns out that 34 of these are always indecomposable, and 5 are always decomposable. The remaining 3 are ambiguous, i.e. each of them has both decomposable and indecomposable geometric realizations.
\end{abstract}

Minkowski sums of polytopes (and more general compact convex sets) occur naturally in various areas of mathematics. It may be of practical interest to determine whether a given polytope can be decomposed into simpler polytopes. Recall that that two polytopes are similar if one can be obtained from the other by a positive scalar multiplication and a translation. A polytope is decomposable if it is equal to a Minkowski sum of two polytopes which are not similar to one another; linguistic logic leads us to describe all other polytopes as indecomposable

In optimization, for example, polytopes are first encountered as the feasible regions for linear programming problems. More general compact convex sets arise as subdifferentials of convex functions. Minkowski sums and decompositions of such sets arise in the definition of quasidifferentiable functions [3]. Most non-smooth optimization algorithms today require the iterative calculation of descent directions. These calculations can be decomposed into simpler ones, if we know that our compact convex set can be decomposed. This is one potential practical application of this topic.

We mention also that Minkowski sums and decompositions have applications in other fields, such as collision detection and robotics [17], pattern recognition [15] and vision geometry [2]. Some computational aspects of these problems are considered in $[11,18,16]$ and the references therein.

This note contributes to the study of decomposability of 3-dimensional polyhedra. In particular, we classify in this sense all polyhedra with 8 vertices and 8 faces. Diagrams of these were first published by Bouwkamp [1, Figures 5 and 8], but they are not labelled in a way which makes them easy to refer to. Instead, we will refer henceforth to Federico [4], who exhibited diagrams of all the 301 combinatorially distinct types of polyhedra with up to 8 faces. On his list, 
those with 8 vertices and 8 faces are numbers 247 to 288 . We present our main theorem now.

THEOREM 1 Of the polyhedra with 8 faces and 8 vertices, 34 types are combinatorially indecomposable, 5 are combinatorially decomposable, and the remaining 3 are ambiguous. More precisely:

(i) Altogether, 22 are indecomposable by virtue of having a strongly connected chain of triangular faces. Specifically, we mean those numbered 247-254, 256, 259-264, 270-272, 278-279, 281 and 286 on the list of Federico.

(ii) A further 10 are indecomposable thanks to a recent result [12, Corollary 10] about non-coplanar 4-cycles, namely numbers 257, 258, 267, 268, 275, 276, 277, 280, 283 and 284.

(iii) Another 2, numbers 269 and 285, are decomposable by a result of Kallay [7, Theorem 10].

(iv) Five types of polyhedra, numbers 255, 265, 266, 273 and 274 are decomposable by a result of Shephard [13, Result (15)].

(v) The remaining 3 cases, numbers 282, 287 and 288, are ambiguous, or conditionally decomposable.

The technical language in this theorem may not be clear to all readers. At the referee's suggestion, we now supply the background material necessary to make the paper self contained. Only after that, will we begin the proof.

The graph, or 1-skeleton, of a polytope is the graph of its edges and vertices. The graph of any polyhedron is isomorphic to a planar graph. This can be seen by projecting the polyhedron onto one of its faces; the resulting graph is called its Schlegel diagram. These planar graphs are the diagrams which appear in [4]. Two polyhedra are called combinatorially equivalent if their graphs are isomorphic. (For higher dimensional polytopes, one needs to consider the lattice of all faces.) We refer to [6, Chapter 3] for further details of these concepts.

A class of combinatorially equivalent polytopes is called combinatorially decomposable if every member of the class is decomposable. Likewise, we define combinatorially indecomposable. If a Schlegel diagram has two different geometric realizations, one decomposable and one indecomposable, that class of polytopes is called ambiguous, or conditionally decomposable.

Basic results about decomposability are due to Gale [5], although he used a different name. It is not hard to check that triangles are indecomposable. Conversely, any polygon with four or more sides is decomposable into a sum of triangles and segments. Here we will mostly consider 3-dimensional polytopes, i.e. polyhedra, although the topic makes sense and is well studied in higher dimensions. Throughout, $V, E$ and $F$ will denote the number of vertices, edges and faces of a given polyhedron.

Gale [5] announced that any pyramid, i.e. the convex hull of a maximal face and a single point, is indecomposable. Clearly a 3-dimensional pyramid has at most one non-triangular face. Since then many authors, beginning with [13], have shown that a polytope is indecomposable if "sufficiently many" of its 2-dimensional faces are triangles. Progessively weaker meanings for "sufficiently many" have been found over time, and we will discuss some of them now. 
Let us say that three vertices form a triangle if they are pairwise adjacent. It is worth noting that a triangle is not necessarily a face. This point was first considered by Kallay [7], who exploited the graph theoretic aspect of this problem. He defined a geometric graph as any graph whose vertices are points in $\mathbf{R}^{d}$ and whose edges are (some of) the line segments joining them. This includes, but is not restricted to, the graphs of polytopes. He then extended the notion of decomposability to such graphs, and showed that a polytope is indecomposable if and only if its edge graph is indecomposable in this sense. We will not repeat this definition here. The following few results are sufficient for our present purposes.

Proposition 2 Any triangle is indecomposable, providing its vertices are not collinear.

Proposition 3 [7, Theorem 8] If two indecomposable geometric graphs have two vertices in common, then their union is indecomposable.

This result motivates the following definition: a sequence $G_{1}, \ldots, G_{k}$ of subgraphs of a graph $G$ will be called a strongly connected chain of graphs if each successive pair has at least two vertices in common. A common technique in this subject has been to find a strongly connected chain of indecomposable subgraphs. For the examples we present, such chains will be quite short.

The next result built on ideas of Kallay and McMullen [9, Theorem 2]. Indeed, the special case when the subgraph contains every vertex of the polytope follows immediately from [7, Corollary 5 and Lemma 6$]$.

Proposition 4 [12, Theorem 8] A polyhedron is indecomposable if and only if there exists a subgraph of its edge graph which is indecomposable, and whose vertex set meets every face of the polyhedron.

To illustrate the usefulness of considering triangles as well as triangular faces, consider any polyhedron whose Schlegel diagram is that appearing in Figure 1. The triangles $A B C$ and $B C D$ together with the preceding results establish its indecomposability. However $B C D$ is not a face of this polyhedron, and no argument using triangular faces can establish its indecomposability. This example has 8 vertices and 9 faces. Later results in this paper combine to show that no similar example can have fewer faces or fewer vertices.

Note also that there is no chain of indecomposable faces in this example which contains every vertex. Thus McMullen's idea that the indecomposable chain should only touch every face, rather than contain every vertex, is quite useful. Most examples in this paper illustrate this point.

Recall next that a graph $G$ is called a 4 -cycle if it has 4 distinct vertices, which can be ordered as $\left\{x_{1}, x_{2}, x_{3}, x_{4}\right\}$, so that its edge set is just $\left\{\left\{x_{1}, x_{2}\right\},\left\{x_{2}, x_{3}\right\},\left\{x_{3}, x_{4}\right\},\left\{x_{4}, x_{1}\right\}\right\}$.

Proposition 5 [12, Proposition 2] Let $C$ be a 4-cycle whose vertices are not coplanar. Then $C$ is indecomposable. 


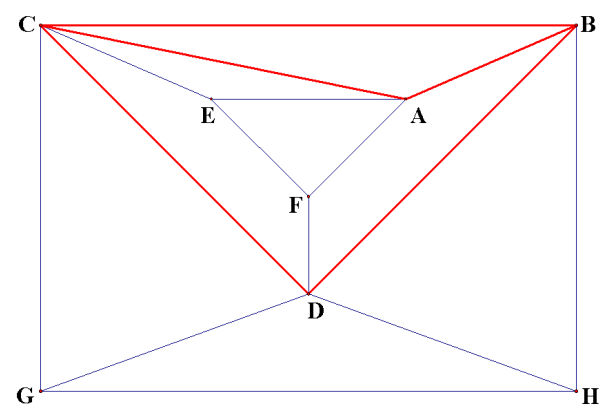

Figure 1: A triangle which is not a face

Likewise it can be shown that any affinely independent cycle in $\mathbf{R}^{d}$ is an indecomposable geometric graph. It is impossible for 5 points in $\mathbf{R}^{3}$ to be affinely independent, so we are really interested only in triangles and 4-cycles. As we have said, the case of triangles has been much studied already. The case of 4-cycles is more interesting to us now.

One of our aims in [12] was to show that that the existence of a noncoplanar 4-cycle could be used to establish indecomposability, in cases where earlier results about triangles were inconclusive. We continue this study here, beginning with the simplest such example, in the sense of having a minimal number of vertices and faces. The indecomposability of the following example is surely known; the point is the easy method of proof.

Proposition 6 There is a combinatorially indecomposable polyhedron with just 7 vertices and 7 faces, but which does not contain a strongly connected sequence of triangles. But it does have an affinely independent 4-cycle, which touches every face.

Proof Let $A=(0,1,0), B=(0,1,1), C=(1,1,0), D=(1,0,0), E=$ $(1 / 2,0,1 / 6), F=(1 / 2,1 / 3,1 / 2), O=(0,0,0)$, and let $P$ be their convex hull. The triangular faces are $A B C, O A B, O D E$ and $D E F$, which clearly cannot be arranged in a strongly connected chain. In any polyhedron combinatorially equivalent to $P$, the 4 -cycle $B C D O$ will be affinely independent, thus ensuring indecomposability.

On the other hand, the following simple result must also be known.

Proposition 7 There is a combinatorially decomposable polyhedron with just 7 vertices and 7 faces.

Proof Just glue a triangular prism and a tetrahedron together. This is clearly the Minkowski sum of a tetrahedron and a line segment. More generally, any polyhedron combinatorially equivalent to this will be decomposable according to $[13$, Result (15)]. 


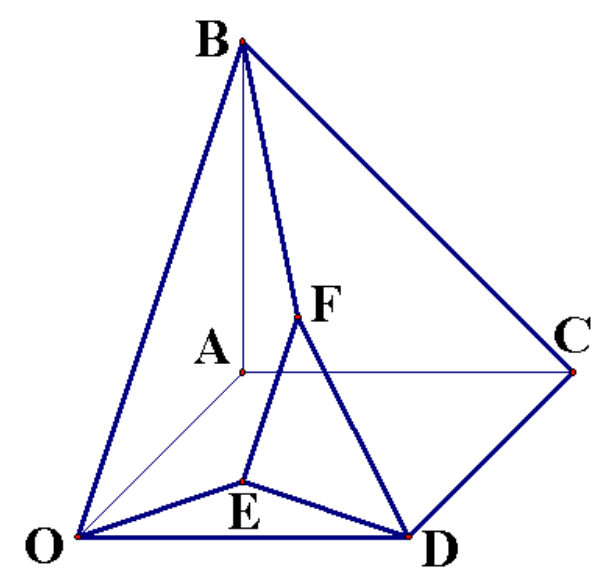

Figure 2: Proposition 6

Euler's relation can be used to establish the symmetric inequalities

$$
V / 2+2 \leq F \leq 2 V-4 .
$$

Smilansky [14, Theorem 6.11(c)] showed that a polyhedron is indecomposable if $F \geq 2 V-6$; this includes the cases when $(V, F)$ is $(4,4),(5,5),(5,6),(6,6)$, $(6,7),(6,8)(7,8)$ or $(7,9)$. He also showed [14, Theorem 6.7] that a polyhedron is decomposable $F<V$; this includes the cases when $(V, F)$ is $(6,5),(7,6)$, $(8,6),(8,7),(9,7)$ or $(9,8)$.

Thus the smallest case for which the number of faces and vertices does not determine decomposability is $V=F=7$. What of the other polyhedra in this class? It is not hard to prove that there are six other combinatorial types, and that they each have a strongly connected chain of triangles which touches every face. Thus they are all indecomposable by classical conditions. This is not new information; it was clearly known to Smilansky $[14, \S 6]$. On Federico's list, they are numbers $19,34,35,38,40$ and 42 . The preceding two examples are numbers 41 and 39 respectively.

Smilansky [14, Corollary 6.8] showed that any indecomposable polyhedron must have at least four triangular faces. This motivated us in [12, Example 13] to exhibit a combinatorially indecomposable polytope with exactly four triangular faces, no two of which had a common edge. Thus traditional methods of proving indecomposability were not available, but the presence of a non-coplanar 4-cycle touching every face saved the day. This seemed like a good example to illustrate our point, but our obsession with making sure that the triangular faces had no common edges forced this example to have nine vertices. It may still be of interest to give other "minimal" examples.

The next smallest case for which the number of faces and vertices does not determine decomposability is clearly $V=F=8$. Smilansky announced that 

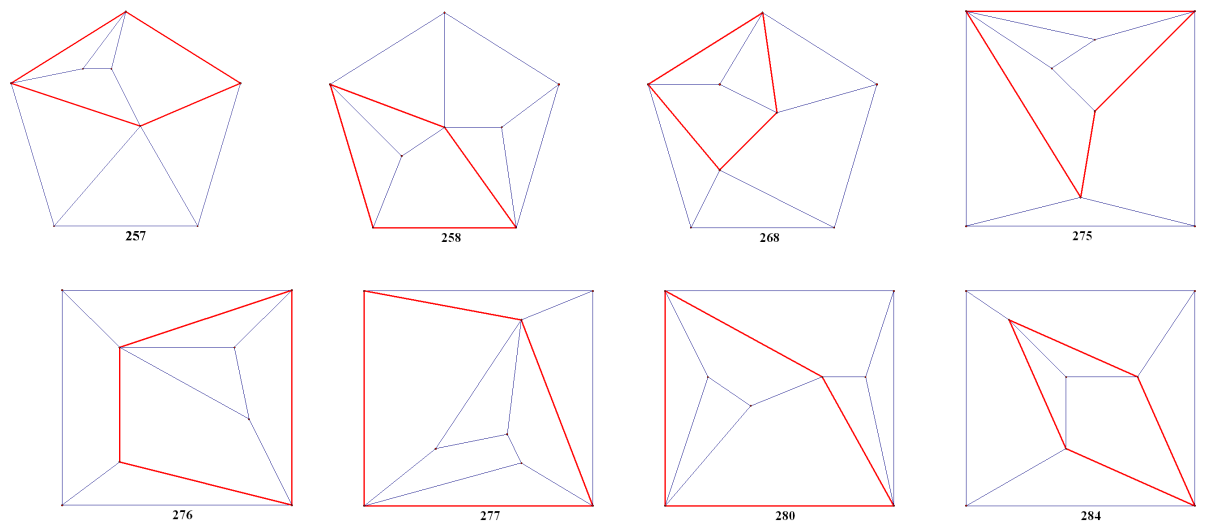

Figure 3: Theorem 1(ii)

some polyhedra in this class are indecomposable, some are decomposable, and some are ambiguous. The rest of this paper is devoted to giving a complete classification for such polyhedra, i.e. to proving Theorem 1. Part (i) is proved simply by examining the numbered diagrams and observing the strongly connected chains of triangular faces.

Proof of Theorem 1 (ii) No polyhedron from these ten classes can be proved indecomposable simply by triangle arguments. However each one contains a non-coplanar 4-cycle, which is indecomposable by Proposition 5. We present here their Schlegel diagrams with an appropriate 4-cycle highlighted.

In each case, three vertices of the indicated 4-cycle belong to one face, whilst the fourth does not. Thus the four vertices cannot be coplanar. In eight of these examples, the 4-cycle touches every face, so indecomposability follows from Proposition 4. For the other two, namely 267 and 283, the 4-cycle fails to touch just one face, but one edge of the 4-cycle lies in a triangle whose other vertex lies in that face. Again, indecomposability is guaranteed, although we need also Propositions 2 and 3.

To decide several of the remaining examples, we need the following result of
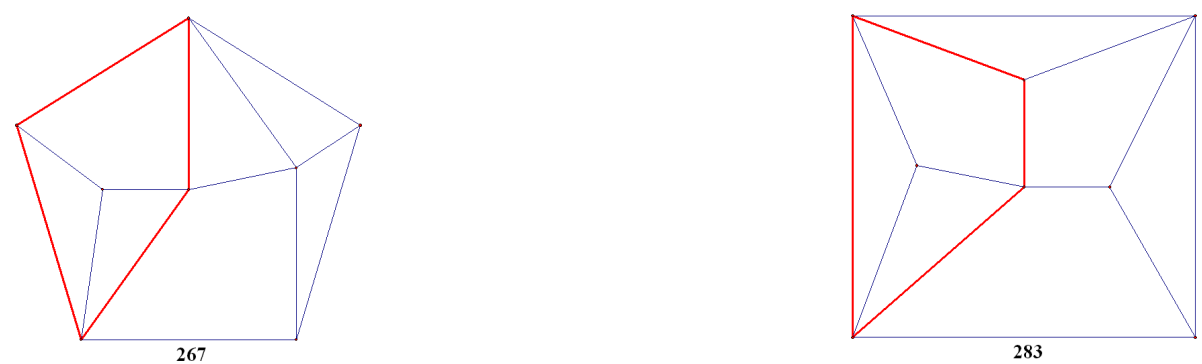

Figure 4: Theorem 1(ii) continued 

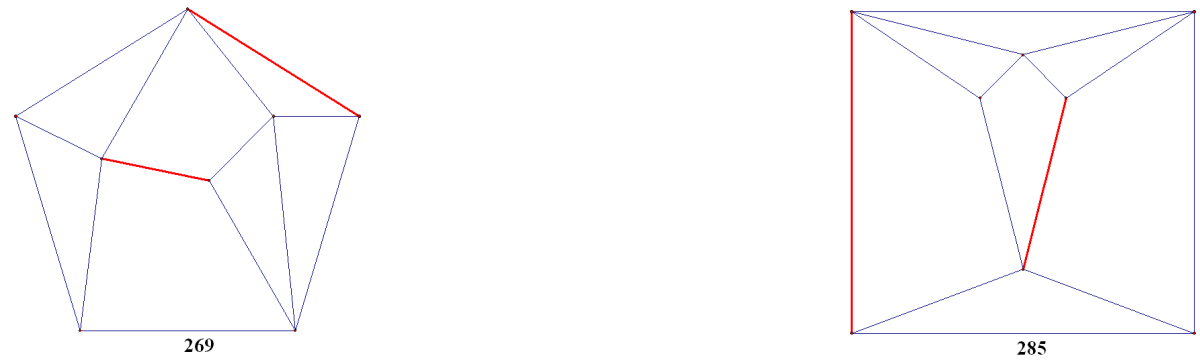

Figure 5: Theorem 1(iii)

Kallay [7, Theorem 10].

THEOREM 8 A geometric graph must be indecomposable if it is the union of two indecomposable subgraphs and two disjoint edges joining them, and the lines containing these edges are skew.

Proposition 5 can also be seen as a special case of Theorem 8 .

Proof of Theorem 1(iii) The polyhedra 269 and 285 are indecomposable by Theorem 8, but not by other arguments. Here are their Schlegel diagrams with an appropriate pair of skew edges highlighted. In each case, the two edges are disjoint, and not cofacial, and yet there is a third edge which is cofacial with both. Thus the two edges cannot be coplanar, i.e. the lines containing them are skew. Strongly connected chains of triangles furnish the required indecomposable subgraphs.

Proof of Theorem 1(iv) Four of these polyhedra, numbers 255, 266, 273 and 274 are obviously decomposable by [13, Result (15)], which implies that a polyhedron is decomposable whenever there is a face in which every vertex is 3-valent, and there are at least two vertices outside this face. So is 265 , pictured here, but this requires a bit more thought. This is clear if the three highlighted edges are parallel; then a line segment will be a summand of the polyhedron. If not, the lines through these edges will be concurrent, and a projective transformation of $\mathbf{R}^{3}$ will send this polyhedron to a combinatorially equivalent one with the three corresponding edges parallel. According to [8], projective transformations preserve decomposability. Alternatively, one can use the argument of [13, Result (15)] to contruct a non-homothetic summand directly.

The remaining three all turn out to be ambiguous. That is, each Schlegel diagram admits one geometric realization which is decomposable, and another (necessarily combinatorially equivalent) which is indecomposable. Number 282 is just [12, Example 11] and will not be discussed again. We will shortly give details for 287 and 288, thereby completing the proof of Theorem 1(v).

The existence of conditionally decomposable polyhedra is not new. Kallay [7] gave an example with ten vertices, and attributed the first example to the thesis of W. J. Meyer. Smilansky [14, Theorem 6.11(b)] announced the existence of an example with just eight vertices and eight faces, referring to his thesis for 


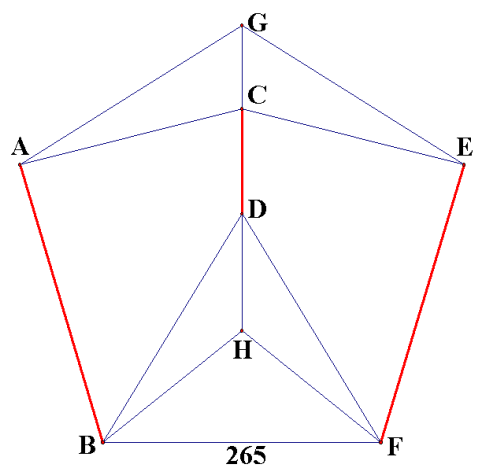

Figure 6: Theorem 1(iv)

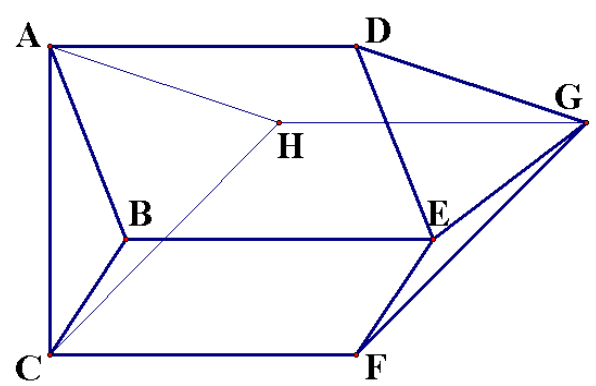

Figure 7: Proposition 9

further details. We have not seen Smilanky's thesis and do not know whether it contains all three examples.

Proposition 9 Example [4, 288] is conditionally decomposable.

Proof Let $P$ be the convex hull of the following points: $A=(-2,0,1), B=$ $(-1,-1,0), C=(-2,0,-1), D=(0,0,1), E=(1,-1,0), F=(0,0,-1)$, $G=(1,1,0)$ and $H=(-1,1,0)$. It is clear from the diagram that $P$ is just the sum of the tetrahedron $D E F G$ and a segment parallel to $(1,0,0)$.

Next we define a combinatorially equivalent polytope $Q$, by perturbing two vertices of $P$. We replace $A$ by $(-2,-\varepsilon, 1+\varepsilon)$ and $B$ by $(-1,-1-\varepsilon, \varepsilon)$ (where $\varepsilon$ need not be so small), and retain the other vertices of $P$. To verify that the labeling induces a one-to-one correspondence between the facial structures of $P$ and $Q$, we just check that the quadrilateral faces remain quadrilateral faces. In detail, $A B D E$ lies in the plane $2 \varepsilon x+(\varepsilon-2) y+(\varepsilon+2) z=2+\varepsilon$, and $C F G H$ has not moved. Moreover, $A D G H$ still lies in the plane $y+z=1$, and $B C E F$ still lies in the parallel plane $y+z=-1$. So $Q$ is combinatorially equivalent to $P$. Since the edges $A D$ and $C F$ lie in parallel planes, but are not parallel, they 


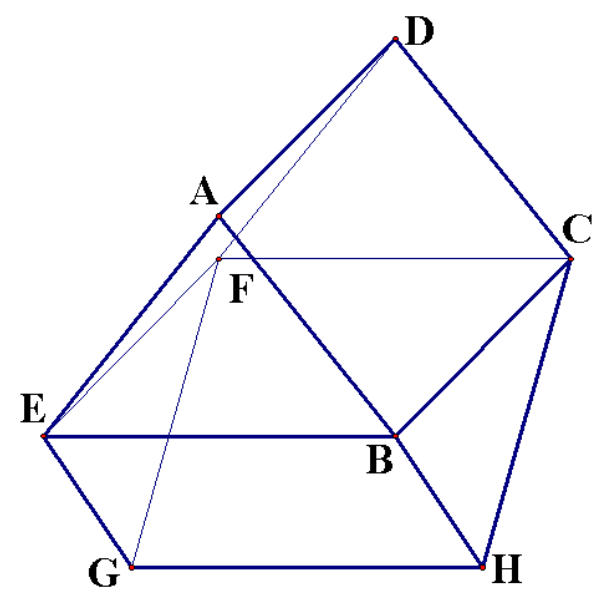

Figure 8: Proposition 10

must be skew. Clearly these edges join the indecomposable subgraphs $A B C H$ and DEFG. By Theorem $8, Q$ is indecomposable.

Proposition 10 Example [4, 287] is conditionally decomposable.

Proof First consider the Minkowski sum of two triangles, one with vertices at $(1,0,0),(-1,0,0)$ and $(0,0, \alpha)$, and the other with vertices at $(0,1,0),(0,-1,0)$ and $(0,0,-\alpha)$, where $\alpha>0$. This decomposable body $P$ can be described as the union of two prisms, with vertices at $A=(0,-1, \alpha), B=(1,-1,0)$, $C=(1,1,0), D=(0,1, \alpha), E=(-1,-1,0), F=(-1,1,0), G=(-1,0,-\alpha)$ and $H=(1,0,-\alpha)$. This has the combinatorial type of [4, 287]. In case $\alpha=\sqrt{3}$, all edges of $P$ have the same length, so this will be a regular body known as the gyrobifastigium. For simplicity, we will now set $\alpha=1$.

Again, we define a combinatorially equivalent polytope $Q$, by perturbing two vertices of $P$. We replace $A$ by $(\varepsilon,-1-2 \varepsilon, 1+\varepsilon)$ and $B$ by $(1,-1-2 \varepsilon, 2 \varepsilon)$, and retain the other vertices of $P$. To check the combinatorial equivalence, note that $A D E F$ still lies in the plane $z-x=1, B E G H$ still lies in the plane $y+z=-1$, and $C F G H$ has not moved. Furthermore $A B C D$ lies in the plane $(1+\varepsilon) x+\varepsilon y+(1+\varepsilon) z=1+2 \varepsilon$ and so remains a face. So $Q$ is combinatorially equivalent to $P$. We note in passing that no two of the four triangular faces have a common edge.

Now, the 4-cycle $B C E F$ is not coplanar, because $C, E$ and $F$ lie in the plane $z=0$ but $B$ does not. Since this 4 -cycle contains an edge from every face, $Q$ is indecomposable.

According to Smilansky [14, p. 46], conditionally decomposable polyhedra with $V$ vertices and $F$ faces exist if and only if $V \leq F \leq 2 V-8$. These inequalities imply either that $V=F=8$, or that $V+F \geq 18$. Thus there are 
no conditionally decomposable polyhedra with 15 edges, but examples with 16 and more edges do exist.

Finally, we consider the higher dimensional case, in order to verify the claim in the first sentence of our abstract.

Proposition 11 All 4-dimensional polytopes with 14 or fewer edges are indecomposable. In dimensions 5 and higher, there are no polytopes with 14 or fewer edges.

Proof The second statement is easy, as every $d$-dimensional polytope has at least $d(d+1) / 2$ edges. For the first, consider a general 4-dimensional polytope. If every 3 -face is a tetrahedron, then every 2 -face is a triangle and indecomposability is clear.

If some 3 -face $F$ is not a tetrahedron, then it has at least 8 edges, and hence there are at most 6 edges outside $F$. Since each vertex has at least 4 neighbors, the existence of two vertices outside $F$ would imply the existence of at least 7 edges outside $F$. This not being so, we conclude that there is only one vertex outside $F$. Then our polytope is a pyramid, and Gale's result implies that it is indecomposable.

\section{References}

[1] Bouwkamp, C.J., 1947. On the dissection of rectangles into squares. (Second communication). Proc. Akad. Wet. Amsterdam, 50, 60-71.

[2] Deguchi, K. and Ghosh, P. K., 2005, Decomposability and indecomposability of binary shapes: a theory of shape arithmetics. Interdisciplinary Information Sciences, 11, 205-213.

[3] Demyanov, V. F. and Rubinov, A. M., 1980, Quasidifferentiable functionals (Russian). Dokl. Akad. Nauk SSSR, 250, 21-25.

[4] Federico, P. J., 1975, Polyhedra with 4 to 8 faces. Geometriae Dedicata, 3, 469-481.

[5] Gale, D., 1954, Irreducible Convex Sets. In: Proc. International Congress of Mathematicians (Amsterdam), vol. 2, pp. 217-218.

[6] Grünbaum, B., 2003, Convex polytopes. Second edition. Graduate Texts in Mathematics, 221. (New York: Springer-Verlag).

[7] Kallay, M., 1982, Indecomposable Polytopes. Israel J. Math., 41, 235-243.

[8] Kallay, M., 1984, Decomposability of polytopes is a projective invariant. In: Convexity and graph theory (Jerusalem, 1981), (Amsterdam: NorthHolland Math. Stud. 87), pp. 191-196.

[9] McMullen, P., 1987, Indecomposable Convex Polytopes. Israel J. Math., 58, 321-323. 
[10] Meyer, W. J., 1974, Indecomposable Polytopes. Trans. Amer. Math. Soc., 190, $77-86$.

[11] D. Pallaschke and J. Rosenmüller, Computing the Minkowski sum of prisms. J. Global Optim., 35 (2006), no. 2, 321-341.

[12] Przesławski, K. and Yost, D., 2007, Decomposability of polytopes. Disc. Comp. Geom., to appear.

[13] Shephard, G. C., 1963, Decomposable Convex Polytopes. Mathematika, 10, $89-95$.

[14] Smilansky, Z., 1987, Decomposability of Polytopes and Polyhedra. Geom. Dedicata, 24, 29-49.

[15] Tuzikov, A.V., Roerdink, J.B.T.M. and Heijmans, H.J.A.M., 2000, Similarity measures for convex polyhedra based on Minkowski addition, Pattern Recognition, 33, 979-995.

[16] Varadhan, G. and Manocha, D., 2006, Accurate Minkowski sum approximation of polyhedral models, Graphical Models, 68, 343-355.

[17] Wein, R.; van den Berg, J. P. and Halperin, D., 2007, The visibility-Voronoi complex and its applications. Comput. Geom., 36, no. 1, 66-87.

[18] Wu, Y.Y., Shah, J.J. and Davidson, J.K., 2003, Improvements to algorithms for computing the Minkowski sum of 3-polytopes. Computer-Aided Design, 35, 1181-1192. 